Organisation of postnatal care affects parents' confidence and security following hospital discharge at 72 hours or less

\title{
Jenny McLeish
}

Policy Research Unit in Maternal Health and Care, National Perinatal Epidemiology Unit, University of Oxford, Old Road Campus, Headington, Oxford OX1 2JD,

UK; jenny.mcleish@npeu.ox.ac.uk

Commentary on: Nilsson I, Danbjørg DB, Aagaard H, Strandberg-Larsen K, et al. Parental experiences of early postnatal discharge: A meta-synthesis. Midwifery 2015; 31(10):926-34.

\section{Implications for practice and research}

- The way health professionals support parents can affect parents' feelings of security and confidence in their role following early discharge.

- Health professionals need to do more to enable fathers to feel included in hospital.

- Future research could explore whether parents' experiences differ according to parity, cultural background, or family structure.

\section{Context}

In many Western countries the period of hospitalisation after birth is now 72 hours or less, and the risks and benefits of this "early" discharge have been debated [1]. Becoming a parent is a time of significant social and emotional change and many new parents experience a lack of confidence in the early days after birth. They may feel both unprepared for, and overwhelmed by, the responsibilities of parenthood [2]. However, this growing body of evidence on parents' needs has not yet affected the organisation of postnatal care in some countries.

\section{Methods}


This meta-analysis was a synthesis of the findings of 10 qualitative studies carried out in Scandinavia, the USA and Australia from 2003 to 2013, reporting parents' experiences of early discharge with a healthy term baby. Having carried out a literature search for studies in Western countries to ensure reasonable homogeneity, the authors used the techniques of meta-ethnography to critically appraise potential studies and then to pool and analyse their findings. They identified the key concepts of each study and translated the findings into one another (looking for areas of common ground while respecting the individual findings). Concepts and categories were integrated to develop final themes. The ten included studies had 237 participants, of whom 150 were mothers, 37 were fathers and 50 unknown; 68 were first time parents, 47 multiparous and parity was not reported for 122 .

\section{Findings}

There were four overlapping themes: 'Feeling and taking responsibility', 'A time of insecurity', 'Being together as a family', and 'Striving to become confident'. Early discharge could increase feelings of insecurity if parents did not feel ready to care for their baby without professional guidance or they were unable to influence the timing of discharge, but could enhance feelings of security if parents were able to develop their confidence though succeeding at parenting tasks and having access to the support they needed. Some fathers felt that they were not able to take responsibility or function as a family until after discharge.

\section{Commentary}

By bringing together the findings of ten qualitative studies in a meta-analysis, this study offers a more comprehensive account of the experiences of new parents in relation to feelings of security and insecurity following what the authors call "early" postnatal discharge (although in some settings a stay of 72 hours would be considered normal or late discharge [2]). The results are consistent with earlier findings that fathers can feel excluded during postnatal care in hospital [3], and that early postnatal discharge is associated with increased fathers' involvement in childcare [4]. 
There is evidence from trials that early discharge is not associated with increased maternal mental health problems provided there is some home visiting support [2], but this study adds depth and breadth to that evidence by exploring the mechanisms by which parents feel their postnatal confidence is built or undermined. The authors usefully frame their findings within Bandura's concept of self-efficacy (5] and the importance of mastery experiences - early discharge had a positive or negative effect according to whether or not parents had access to the individualised post-discharge information and feedback support from professionals, that they needed to gain confidence in their parenting.

These findings affirm current guidance in England which recommends that the timing of hospital discharge should be discussed with the mother, and that information offered postnatally should aim to empower the mother to take care of her baby [6]. Because of the limited demographic reporting, this study does not, however, enable conclusions to be drawn about whether the findings might be affected by parity, partnership status, cultural background, age, education or socio-economic position.

\section{References}

1. Brown S, Small R, Faber B, et al. Early postnatal discharge from hospital for healthy mothers and term infants. Cochrane Database Syst Rev 2002;3:CD002958.

2. Barclay L, Everitt L, Rogan F, et al. Becoming a mother - an analysis of women's experience of early motherhood. Journal of Advanced Nursing 1997;25:719-728.

3. Deave T and Johnson D. The transition to parenthood: what does it mean for fathers? Journal of Advanced Nursing 2008;63(6):626-633

4. Waldenström U. Early and late discharge: fathers' involvement in infant care. Early Human Development 1988;17:19-28.

5. Bandura, A. Self-efficacy: The Exercise of Control. New York: W.H.Freeman \& co., 1997

6. National Institute for Health and Clinical Excellence. Postnatal care up to 8 weeks after birth: clinical guideline. London: NICE, 2006 